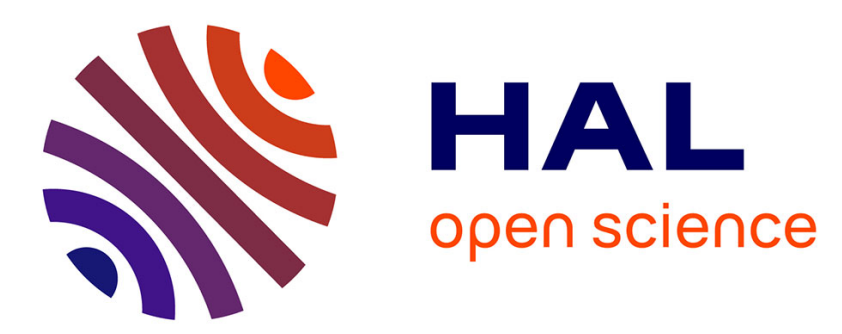

\title{
Elimination de cones d'ombre acoustique par combinaison d'images échographiques mode B
}

\author{
A. Hernandez, O. Basset, P. Chirossel, G. Gimenez
}

\section{To cite this version:}

A. Hernandez, O. Basset, P. Chirossel, G. Gimenez. Elimination de cones d'ombre acoustique par combinaison d'images échographiques mode B. Journal de Physique IV Proceedings, 1994, 04 (C5), pp.C5-1301-C5-1304. 10.1051/jp4:19945289 . jpa-00253001

\section{HAL Id: jpa-00253001 https://hal.science/jpa-00253001}

Submitted on 1 Jan 1994

HAL is a multi-disciplinary open access archive for the deposit and dissemination of scientific research documents, whether they are published or not. The documents may come from teaching and research institutions in France or abroad, or from public or private research centers.
L'archive ouverte pluridisciplinaire HAL, est destinée au dépôt et à la diffusion de documents scientifiques de niveau recherche, publiés ou non, émanant des établissements d'enseignement et de recherche français ou étrangers, des laboratoires publics ou privés. 


\title{
Elimination de cones d'ombre acoustique par combinaison d'images échographiques mode B
}

\section{A. HERNANDEZ, O. BASSET, P. CHIROSSEL* et G. GIMENEZ}

Laboratoire de Traitement du Signal et Ultrasons, URA 1216 du CNRS, INSA Lyon, bât. 502, 20 avenue Albert Einstein, 69621 Villeurbanne cedex, France

* Hôpital Cardio-Vasculaire et Pneumologique Louis Pradel, Service Radiologie, BP. Lyon-Montchat, 69394 Lyon cedex 03, France

\begin{abstract}
RESUME : Un système de reconstruction d'images échographiques a été mis au point dans le but d'éliminer les cônes d'ombre engendrés sur les images par des structures hyperéchogènes. L'image reconstruite est la combinaison de plusieurs vues partielles et complémentaires, obtenues en tournant autour de l'hyperéchogénicité. La position relative des images est donnée par un bras articulé de localisation à l'extrêmité duquel est fixée la sonde échographique.
\end{abstract}

\begin{abstract}
A system devoted to echographic image reconstruction has been performed in order to eliminate acoustic shadowing generated in the images by the hyperechogenic structures. The image reconstruction consists in the combination of several complementary and partial views, obtained by turning around the hyperechogenicity. The relative position of the images is given by a pantograph localisation system on which is fixed the echographic transducer.
\end{abstract}

\section{1 - INTRODUCTION}

Lorsqu'un faisceau ultrasonore explore un milieu contenant une structure d'impédance très différente de celle du milieu, l'interface milieu/structure réfléchit très fortement l'énergie ultrasonore incidente. Une telle structure est dite hyperéchogène. Sa présence se manifeste le plus souvent sur l'image échographique mode B par un cône d'ombre précédé d'un trait blanc. Le cône d'ombre correspond à la zone où les ultrasons ne pénètrent pas, tandis que le trait blanc matérialise l'interface réfléchissante (fig. 1).
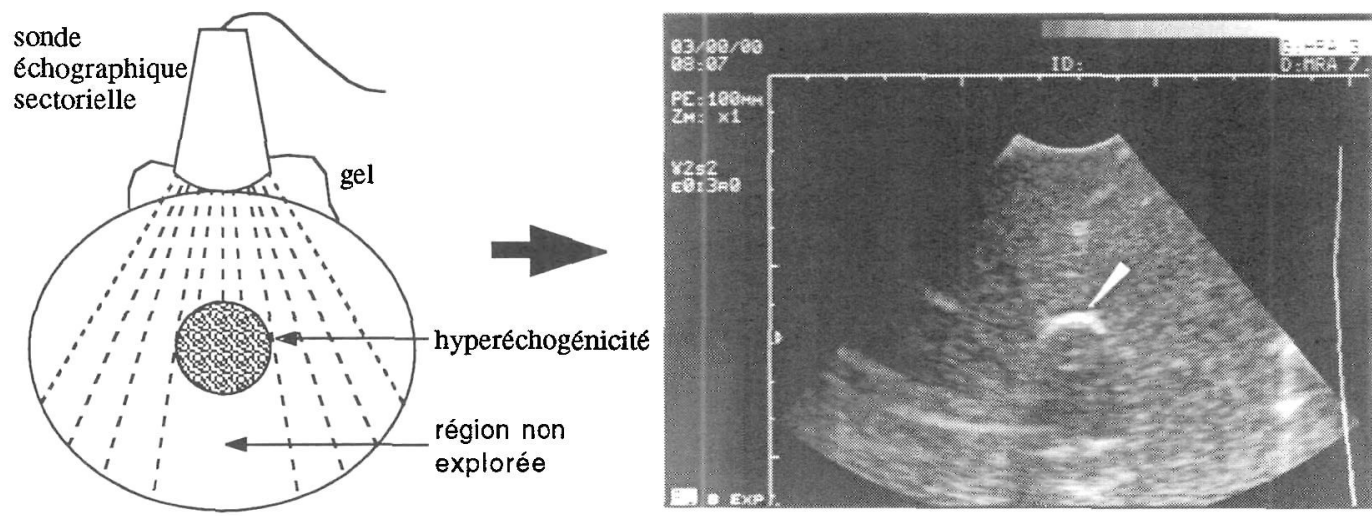

Figure 1 - Représentation sur une image échographique du cône d'ombre généré par une structure hyperéchogène. La flêche blanche indique la position de l'interface réfléchissante. 
Les informations relatives à la zone d'ombre ne sont accessibles que si l'on explore le milieu sous des incidences différentes. Dans les images ainsi obtenues, de nouveaux cônes d'ombre apparaissent suivant les nouvelles directions de propagation ultrasonore. Ainsi, chacune des images échographiques restitue une information partielle, mais complémentaire, sur les contours de la structure hyperéchogène et sur le milieu environnant.

Les contours de la structure hyperéchogène et le milieu qui l'entoure peuvent être reconstruits sur une même image sans ombre, ni artéfact par tomographie ultrasonore [1]. Toutefois, cette technique reste difficile à mettre en oeuvre, notamment à cause de la complexité des algorithmes de reconstruction [2]. Lorsque la qualité de l'image n'est pas le facteur déterminant recherché pour l'image reconstruite, il est préférable d'utiliser une technique de superposition d'images échographiques mode B. L'objet de ce travail est le développement d'une telle méthode permettant la reconstruction d'une image complète à partir de plusieurs images partielles complémentaires.

\section{2 - PRINCIPE}

La mise en place de cette technique nécessite la connaissance de la position relative dans l'espace, des images partielles par rapport à un repère fixe. De plus ces images doivent être acquises dans un même plan de coupe de l'objet. Ces deux contraintes ont été résolues grâce à l'utilisation d'un bras articulé de repérage (ou bras de localisation).

Le bras de repérage est composé de plusieurs segments reliés entre eux par six articulations. Le premier segment est fixé sur un support qui reste immobile dans le repère du laboratoire. L'autre extrêmité du bras est mobile et peut se mouvoir dans un volume important tout autour du support grâce aux six degrés de liberté que lui confèrent les articulations. Ce dernier segment du bras de localisation permet la fixation de la sonde échographique. La connaissance de la longueur des différents segments ainsi que les valeurs angulaires imprimées à chacune des articulations permettent de calculer les coordonnées de la sonde en faisant six changements de repère successifs.

Un potentiomètre est placé dans chacune des six articulations. Celui-ci renvoie instantanément une tension électrique proportionnelle à l'angle de rotation imposé à l'articulation associée. Les tensions électriques en provenance des six articulations sont ensuite converties en signaux numériques grâce à un convertisseur analogique numérique (C.A.N.). Un micro-ordinateur recoit ces signaux numériques, porteurs des informations angulaires par l'intermédiaire d'une carte d'acquisition (NAUTIL LOGIC 40). Un algorithme permet alors de calculer la position et l'orientation de la sonde échographique, et par là même celle des images. Ce programme permet également le stockage des coordonnées spatiales de la sonde dans un fichier et l'affichage d'informations sur l'écran du PC qui vont aider l'opérateur, lors de l'acquisition des images, à maintenir la sonde échographique dans un même plan d'exploration.

Les différentes images acquises par l'échographe sont enregistrées sur la bande vidéo d'un magnétoscope. Simultanément l'acquisition des coordonnées de ces images est obtenue au moyen du bras de repérage. La chaine d'acquisition est schématisée sur la figure 2 . Les images échographiques sont ensuite numérisées (carte MATROX PIP 1024) afin d'être traitées par ordinateur.

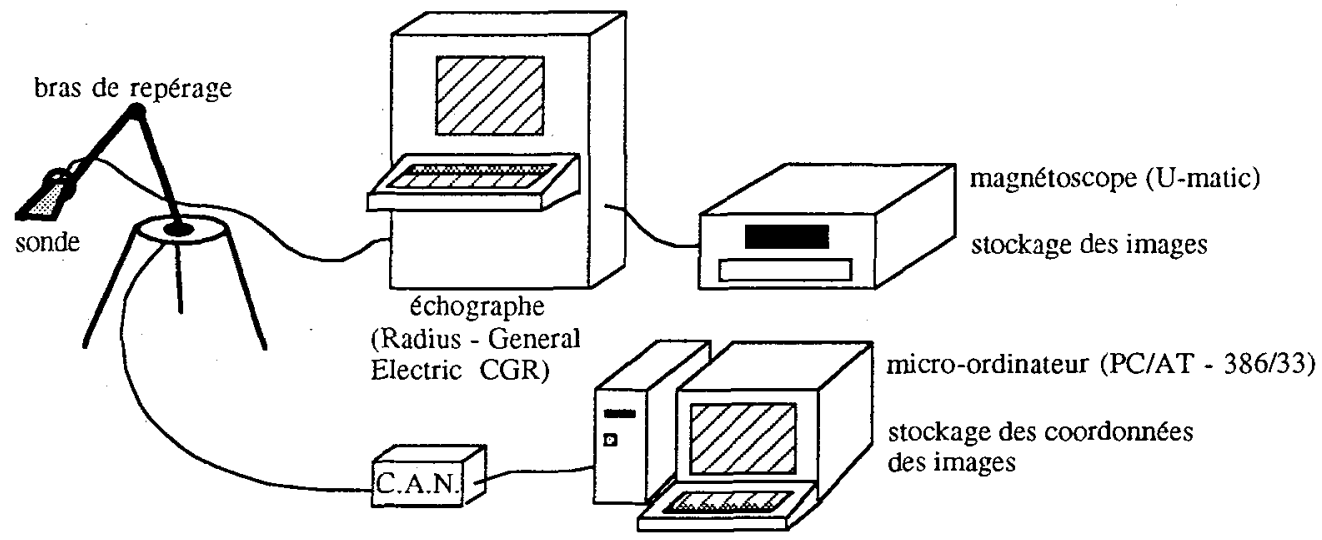

Figure 2 - Chaîne d'acquisition des images et de leurs coordonnées spatiales.

A partir des images partielles complémentaires et de leurs coordonnées respectives dans l'espace d'acquisition, une image complète sans cônes d'ombre, peut alors être reconstruite informatiquement.

La première étape de la reconstruction consiste à mettre en correspondance les images partielles d'un 
même plan de coupe. Ces images subissent des rotations et des translations (pixel par pixel) par rapport à l'une d'entre elles qui est choisie comme référence. Les paramètres de rotation et de translation sont calculés à partir du fichier contenant la position et l'orientation des images échographiques.

La deuxième étape de la reconstruction consiste à superposer les images partielles mises en correspondance pour obtenir l'image finale reconstruite. Deux methodes sont envisageables : 1) on fait la moyenne en niveau de gris des différentes images; 2) on affecte à un pixel donné de l'image finale, le niveau de gris le plus élevé des pixels correspondants sur les images partielles. La première méthode permet d'atténuer le bruit; la deuxième méthode permet d'améliorer le contraste dans l'image de reconstruction.

\section{3 - EXPERIMENTATIONS}

Un fantôme en agar-agar (gélatine dans laquelle les caractéristiques de propagation des utrasons sont sensiblement les mêmes que celles dans les tissus vivants) a été utilisé pour tester la méthode de reconstruction. Ce fantôme est un bloc cubique traversé par un puits de section ovale et contenant de l'air. Trois images échographiques (fíg. 3-a, 3-b et 3-c) ont été réalisées dans un même plan sur trois des faces du fantôme, de manière à obtenir des vues de coupe de la colonne d'air contenue dans celui-ci. L'air étant hyperéchogène vis à vis de l'agar-agar, il en résulte que des cônes d'ombre apparaissent dans ces images. Les coordonnées des images obtenues grâce au bras de repérage durant la phase d'acquisition permettent alors la reconstruction d'une image sans cônes d'ombre (fig. 3-d). La continuité des contours y est respectée. La forme et les dimensions de l'ovale y sont conformes à celles mesurées sur la section du bloc d'agar-agar.

Cette méthode de reconstruction a ensuite été appliquée dans le domaine médical à des régions anatomiques hyperéchogènes comme les os longs et la colonne d'air du pharynx.

En ce qui concerne le pharynx, une image de coupe visualisant sa circonférence pourrait aider à diagnostiquer plus facilement une maladie respiratoire : le syndrome des apnées du sommeil [3]. En effet, cette maladie, qui se caractérise par de nombreuses pauses respiratoires durant le sommeil, résuite dans la majorité des cas d'un affaissement des parois du pharynx. Malheureusement, les résultats obtenus pour la reconstruction des contours pharyngés sont peu satisfaisants. Dans le meilleur des cas, on reconstitue seulement la moitié de la circonférence du pharynx. La principale raison de cet échec est imputable aux structures osseuses : maxillaire inférieur, cervicales et os hyoïde, qui limitent la fenêtre d'ouverture sur le pharynx à l'exploration ultrasonore.

Dans le cas des os longs, on cherche à obtenir une image de la coupe transversale des membres associés à ces os, afin de visualiser sur une seule image les contours osseux et les tissus adjacents. Les images obtenues sont satisfaisantes. On reconstitue de manière relativement précise et nette les contours de ces os. La figure 4-c présente une image de reconstruction de la coupe du fémur à partir de deux images partielles complémentaires (fig. 4-a et 4-b).

\section{4 - CONCLUSION}

Une technique de reconstruction échographique permettant d'imager les contours de structures hyperéchogènes a été mise au point. Les images échographiques acquises dans un même plan autour de l'hyperéchogénicité sont repérées dans l'espace grâce à un bras de localisation. Les images sont ensuite numérisées, elles sont mises en correspondance par la connaissance de leurs coordonnées spatiales et enfin, elles sont superposées de manière à donner naissance à l'image de reconstruction.

La technique de repérage et de reconstruction des images a été testée sur fantôme et donne des résultats satisfaisants. L'erreur moyenne engendrée par le bras de localisation sur l'image finale est de $1 \mathrm{~mm}$, ce qui compte tenu de la résolution des images échographiques reste acceptable. Pour obtenir cette précision, certaines précautions s'imposent durant la phase d'acquisition des images. D'une part, l'objet ou le patient sur qui on réalise les images doit rester parfaitement immobile, et d'autre part, les différentes images d'un même plan de coupe doivent être acquises dans une "tranche" la plus étroite possible.

\section{REFERENCES}

1 - SEHGAL (C.M), LEWALLEN (D.G), NICHOLSON (J.A), ROBB (R.A) et GREENLEAF (J.F), Ultrasound transmission and reflection computerized tomography for imaging bones and adjoining soft tissues, IEEE 1988 Ultrasonics Symp. Proc., Chicago, IL, 2-5 Oct. 1988, vol.2, p.849-859.

2 - DENIS (F), Tomographie acoustique d'objets faiblement réfringeants : prise en compte de la réfraction pour la reconstruction d'images de vitesse, Thèse INSA Lyon, 1990.

3 - FERRETTI (G), PEPIN (J.L), VEALE (D), CROUZET (G) et COULOMB (M), Apport de l'imagerie à

l'exploration du syndrome d'apnée obstructive du sommeil, Rev. Im. Med., 1993, vol.5, p.3-13. 


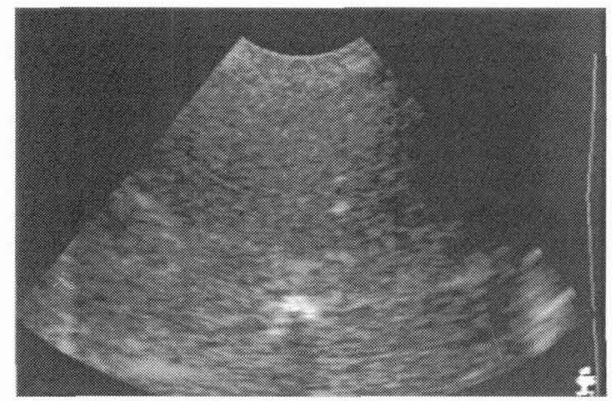

a - image 1

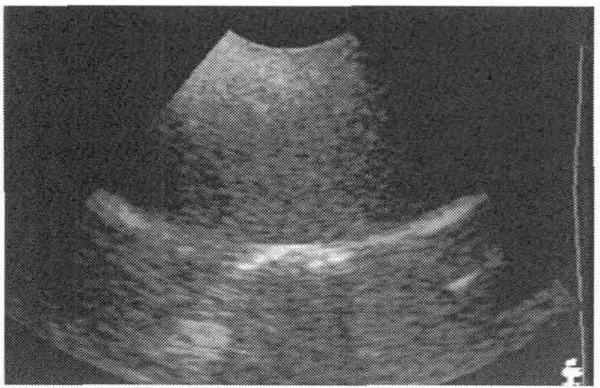

c - image 3

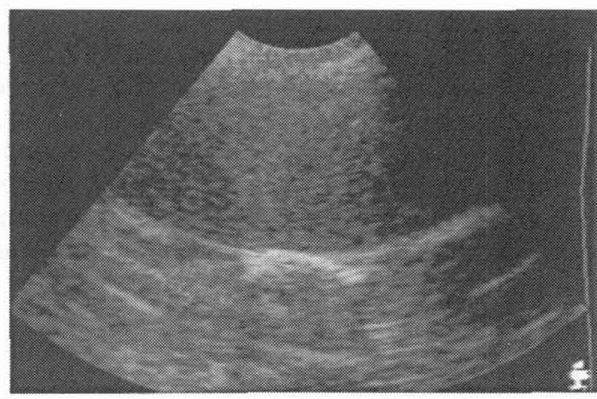

b - image 2

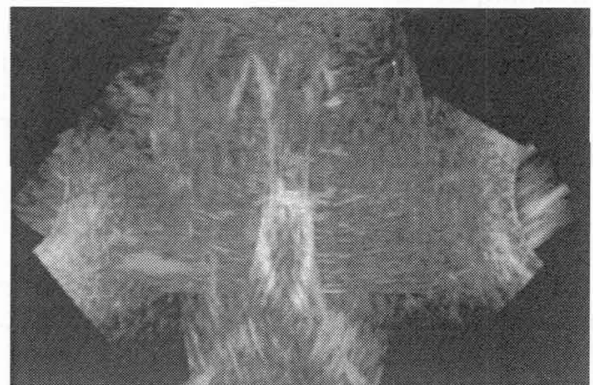

d - image de reconstruction

Figure 3 - Reconstruction d'une image de coupe (d) d'un bloc d'agar-agar contenant une colonne d'air, à partir de trois images échographiques (a,b et $\mathrm{c}$ ).

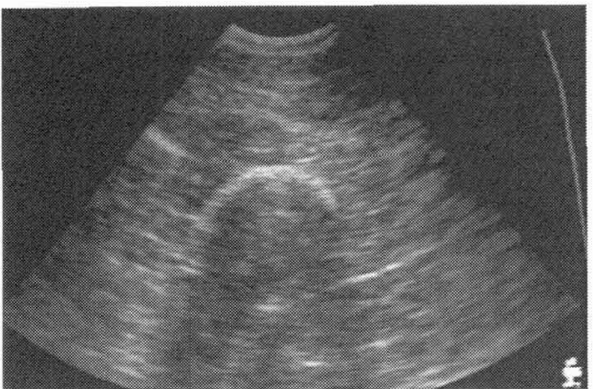

a - image 1

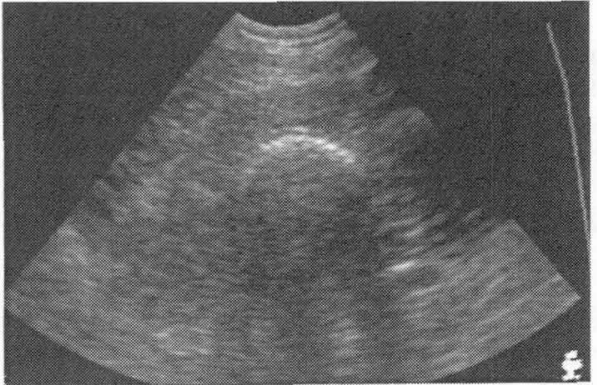

b - image 2

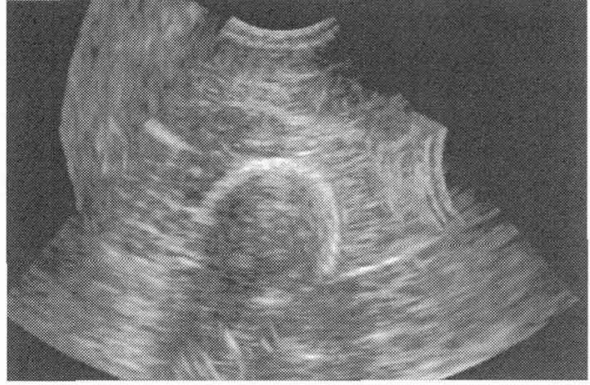

$\mathrm{c}$ - image de reconstruction

Figure 4 - Reconstruction d'une image de coupe (c) des contours du fémur à partir de deux images échographiques ( $a$ et $b$ ). 